\title{
Detrimental Effects and Prevention of Acidic Electrolytes on Oxygen Reduction Reaction Catalytic Performance of Heteroatom-Doped Graphene Catalysts
}

OPEN ACCESS

Edited by:

Dingshan Yu,

Sun Yat-sen University, China

Reviewed by:

Tianhua Zhou,

Chinese Academy of Sciences, China Qiang Wang

Nanjing Tech University, China

${ }^{*}$ Correspondence:

Dong Liu

liudong@mail.buct.edu.cn

Lipeng Zhang

zhanglp@mail.buct.edu.cn

Zhenhai Xia

Zhenhai.xia@unt.edu

Specialty section

This article was submitted to

Energy Materials,

a section of the journal

Frontiers in Materials

Received: 02 August 2019 Accepted: 01 November 2019 Published: 21 November 2019

Citation:

Ma J, Gong L, Shen Y, Sun D, Liu B,

Zhang J, Liu D, Zhang $L$ and Xia Z

(2019) Detrimental Effects and

Prevention of Acidic Electrolytes on Oxygen Reduction Reaction Catalytic Performance of Heteroatom-Doped

Graphene Catalysts.

Front. Mater. 6:294.

doi: 10.3389/fmats.2019.00294

\author{
Jun Ma ${ }^{1}$, Lele Gong ${ }^{1}$, Yang Shen ${ }^{1}$, Defeng Sun ${ }^{1}$, Bowen Liu ${ }^{1}$, Jing Zhang ${ }^{2}$, Dong Liu ${ }^{1 *}$, \\ Lipeng Zhang ${ }^{1 *}$ and Zhenhai Xia ${ }^{3 *}$ \\ ${ }^{1}$ College of Chemical Engineering, Beijing University of Chemical Technology, Beijing, China, ${ }^{2}$ College of Materials Science \\ and Engineering, Northwestern Polytechnical University, Xi'an, China, ${ }^{3}$ Department of Materials Science and Engineering, \\ University of North Texas, Denton, TX, United States
}

Heteroatom-doped carbon based catalysts have been demonstrated as one of the most promising electrocatalysts to replace traditional noble metal catalysts, such as Pt, for oxygen reduction reaction (ORR) in proton-exchange membrane fuel cells (PEMFCs). However, experimental results have shown that the carbon based catalysts exhibit inferior catalytic activities in acidic than in alkaline mediums. As the catalytic mechanism is unclear, there is no effective strategy to design and synthesize highly efficient carbon based catalysts working in acidic medium. In this work, the density functional theory (DFT) methods were applied to understand the inferior performance of doped graphene in acid. Our results show that the excellent performance of doped graphene is downgraded by protonation of dopants and the adsorption of acidic anions. The calculated ORR overpotentials were increased due to the protonation and the aggregation of acid anions on the graphene surface. To enhance the catalytic activities, the adverse effects of protonation and acid anions should be minimized as much as possible. These insights provide a direction to boost the catalytic efficiency and stability of metal-free carbon based catalysts for clean energy conversions and storages.

Keywords: doping graphene nanoribbons, oxygen reduction reaction, catalytic activity, acidic medium, DFT simulation

\section{INTRODUCTION}

Proton-Exchange Membrane Fuel cells (PEMFCs), as sustainable and promising energy conversion devices, have attracted widely attention in energy applications owning to their high efficiency and no pollution (Stephens et al., 2016). In PEMFCs, the key reaction, oxygen reduction reaction (ORR) is sluggish and requires highly efficient catalysts (Debe, 2012; Zhang G. et al., 2019). Generally, noble metals such as Pt, have been used to boost the ORR (Shao et al., 2016). However, the high cost and the scarcity of noble metals hinder the large-scale commercial application of PEMFCs. Recently, great advances in metal-free carbon catalysts for ORR endowed new possibilities for the development of PEMFCs (Dai et al., 2015; Liu and Dai, 2016; Hu and Dai, 2019; Zhao et al., 2019). It has been demonstrated that the catalytic efficiency of heteroatom doped graphene for ORR are comparable to that of Pt in alkaline medium, and these dopants 
include N (Gong et al., 2009; Wang et al., 2018a) B (Yang et al., 2011), P (Zhang et al., 2013; Wu et al., 2015) and so on. However, in acidic medium, the performance of doping carbon cannot meet the commercial demand in PEMFCs (Yang et al., 2019). For example, $\mathrm{N}$ doped ultrathin carbon nanosheets showed an onset potential of 0.95 and $0.78 \mathrm{~V}$ in $0.1 \mathrm{M} \mathrm{KOH}$ and $0.5 \mathrm{M} \mathrm{H}_{2} \mathrm{SO}_{4}$, respectively (Jiang et al., 2019). Similarly, the change of $\mathrm{pH}$ from $12-14$ to $0-2$ leaded to a significant degradation of the catalytic activity during electrochemical testing (Wan et al., 2015). It was unclear why the same catalysts showed different catalytic activities in different electrolyte environments (Zeradjanin, 2018). These experimental observations have not been completely understood in terms of their catalytic mechanisms on the surface of catalysts.

Density functional theory (DFT) simulation is an effective theoretical approach to study the ORR mechanisms (Kulkarni et al., 2018; Wang et al., 2018b; Zhang L. et al., 2019). The direct interactions between the surface of catalysts and the intermediate radicals of ORR in vacuum have been broadly studied by the simulation methods (Li et al., 2017; Xue et al., 2018; Yang et al., 2018). For instance, Zhao et al. (2015) discovered that the most desirable active sites on $\mathrm{X}$-doped (X denotes to the elements in the $\mathrm{p}$ block of the periodic table) graphene originate from the optimal adsorption energies of intermediates of $\mathrm{OOH}^{*}, \mathrm{O}^{*}$, and $\mathrm{OH}^{*}$. However, due to the neglect of electrolyte environment in simulation, the issue of $\mathrm{pH}$-dependence of the catalytic activity has not yet to be well-addressed in these limited models. As we know, the surface of catalysts contacts with electrolyte containing various anions and cations. In the process of evaluating the activity of the catalysts, the effects of these ions cannot be completely ignored (Yang et al., 2019).

In this work, we evaluated the effect of various solution composition of electrolytes to explicitly clarify the distinction of catalytic behaviors in acid and base conditions. We found there were two factors, protonation and adsorption of acid radical ions, which resulted in the inferior catalytic performance of the doped graphene in acidic environments. The insights provide a direction and hints to rationally design and optimize high performance carbon-based catalysts for PEMFCs.

\section{COMPUTATIONAL METHODS AND MODELS}

In this work, all the first-principles calculations were implemented in the plane wave Vienna Ab-initio Simulation Package (VASP) code with the framework of DFT (Kresse and Furthmüller, 1996a,b). The projector augmented wave (PAW) pseudo-potentials method (Kresse and Joubert, 1999) was used to describe the core-electron interactions. The parameterization of the electronic exchange and correlation effects were realized by the Perdew-Burke-Ernzerh (PBE) method (Perdew et al., 1996) within the Generalized Gradient Approximation (GGA). The core-valence electrons configurations corresponding to elements in this paper are as follows: $\mathrm{H}-1 \mathrm{~s}^{1}, \mathrm{C}-[\mathrm{He}] 2 \mathrm{~s}^{2} 2 \mathrm{p}^{2}, \mathrm{O}-[\mathrm{He}] 2 \mathrm{~s}^{2} 2 \mathrm{p}^{4}$, B- $[\mathrm{He}] 2 \mathrm{~s}^{2} 2 \mathrm{p}^{1}, \quad \mathrm{~N}-[\mathrm{He}] 2 \mathrm{~s}^{2} 2 \mathrm{p}^{3}, \quad \mathrm{P}-[\mathrm{Ne}] 3 \mathrm{~s}^{2} 3 \mathrm{p}^{3}, \quad \mathrm{~S}-[\mathrm{Ne}] 3 \mathrm{~s}^{2} 3 \mathrm{p}^{4}$, $\mathrm{Cl}-[\mathrm{Ne}] 3 \mathrm{~s}^{2} 3 \mathrm{p}^{5}$ (Zhu et al., 2019). The cutoff energy was selected to be $500 \mathrm{eV}$ and a $4 \times 4 \times 1$ grid of K-point sampling was generated by Monkhorst-Pack Scheme. The structures were relaxed until the energy and the force converging to $1 \times 10^{-4} \mathrm{eV}$ and $0.01 \mathrm{eV} / \AA$, respectively.

All built models in this work are based on doped graphene nanoribbons (GR) with zigzag edges. The doped heteroatoms $(\mathrm{X}=\mathrm{N}, \mathrm{B}, \mathrm{P})$ locating at the zigzag edges are favorable to boost the ORR catalytic activity (Jiang et al., 2007; Li et al., 2014; He et al., 2016). We mainly focus on nitrogen-doped graphene with pyridinic-N, which is considered to be the origin of catalytic activity (Li et al., 2014; Guo et al., 2016; Zhang L. et al., 2019). The GRs were constructed with periodic boundary condition in $\mathrm{x}$-direction. The width $(\mathrm{W})$ of the nanoribbons is 5 rings $(\sim 12$ $\AA$ ) due to the adsorption energy of the intermediates at the GR edge no longer change as the width further increased, as shown in Figure 1A. To avoid the interaction between slabs, a vacuum
A

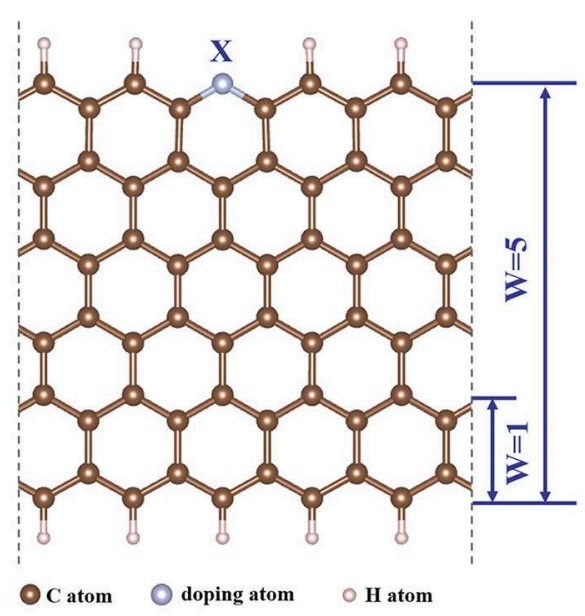

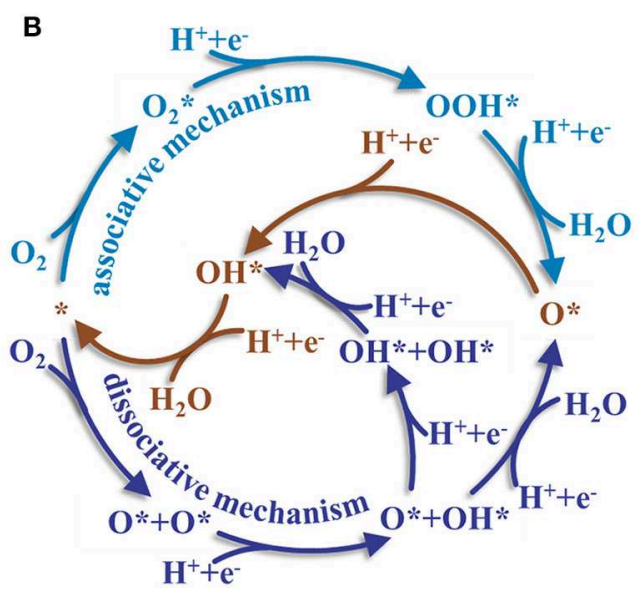

FIGURE 1 | (A) The zigzag heteroatom doped graphene nanoribbons utilized in the calculations. (B) The schematic of possible elementary reaction pathways for the ORR within associative and dissociative mechanisms. 
spacing was added with the value of 11 and $15 \AA$ in the $y$ - and z-directions, respectively. In addition, Deng et al. (2016) claimed that the $\mathrm{C}$ atoms near $\mathrm{N}$-dopant exhibited catalytic activity for ORR. In our work, the potential catalytic active sites near the doped $\mathrm{N}$ were numbered as shown in the inset of Figure 2. The GR models are referred with the following format:

$$
\mathrm{X}-\mathrm{GR} / m \mathrm{Y} / n \mathrm{Z},
$$

where $\mathrm{X}, m \mathrm{Y}$, and $n \mathrm{Z}$ represent doping element $(\mathrm{N}, \mathrm{B}$, $\mathrm{P})$, absorption of $m$ protons or hydroxides and absorption of $n$ anions $\left(\mathrm{ClO}_{4}^{-*}, \mathrm{HSO}_{4}^{-*}\right.$ and $\left.\mathrm{SO}_{4}^{2-*}\right)$, respectively. For example, $\mathrm{N}-\mathrm{GR} / 2 \mathrm{H} / 2 \mathrm{ClO}_{4}^{-}$denotes nitrogen-doped graphene nanoribbons adsorbed with 2 protons and $2 \mathrm{ClO}_{4}^{-}$groups.

In principle ORR occurs via two pathways: two-electron and four-electron transfer; the latter one is recognized to be more efficient than the former one (Lu et al., 2019). Consequently, we calculated all possibly four-electron transfer pathways (Figure 1B), including associative and dissociative mechanisms with different configurations of capturing $\mathrm{O}_{2}$ (Yang et al., 2017; Ji et al., 2018). The asterisk stands for the adsorption of intermediates at active sites of doped GR. We employed the following method proposed by Nørskov to describe the reaction Gibbs free energy $(G)$ of the sub-reactions. The change of $G$ between the initial and final states for each elementary step is expressed by the following equation (Man et al., 2011):

$$
\Delta \mathrm{G}=\Delta \mathrm{E}_{\mathrm{DFT}}+\Delta \mathrm{E}_{\mathrm{ZPE}} \mathrm{T} \Delta \mathrm{S}+\Delta \mathrm{G}_{\mathrm{U}}+\Delta \mathrm{G}_{\mathrm{pH}}
$$

where $\Delta \mathrm{E}_{\mathrm{DFT}}, \Delta \mathrm{E}_{\mathrm{ZPE}}, \mathrm{T}, \Delta \mathrm{S}, \Delta \mathrm{G}_{\mathrm{U}}$, and $\Delta \mathrm{G}_{\mathrm{pH}}$ are the electronic energy difference obtained from DFT calculations, the change of zero-point energy, the temperature ( $298 \mathrm{~K}$ ), the change of entropy, the change of free energy due to applied potential on

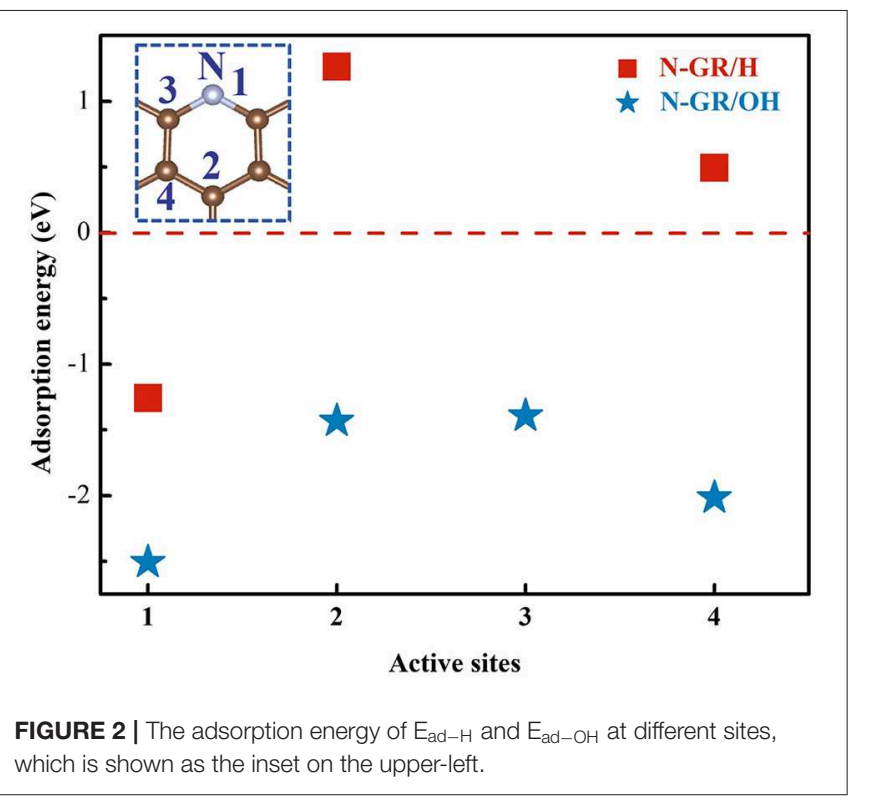

electrode, and the corrected change of free energy effected by the acidity and alkalinity of the solution.

$$
\begin{aligned}
\Delta \mathrm{G}_{\mathrm{U}} & =e \mathrm{U} \\
\Delta \mathrm{G}_{\mathrm{pH}} & =k_{\mathrm{B}} \operatorname{TIn}\left[\mathrm{H}^{+}\right]
\end{aligned}
$$

where $e, \mathrm{U}$, and $k_{\mathrm{B}}$ stand for the transferred charge, the potential at the electrode and Boltzmann constant.

Besides, at standard hydrogen electrode $(\mathrm{U}=0, \mathrm{pH}=0$, pressure $=1$ bar and temperature $=298 \mathrm{~K}$ ), the potential of an electron-proton pair $\left(\mathrm{H}^{+}+\mathrm{e}^{-}\right)$was substituted by the half of the free energy of the hydrogen $\left(1 / 2 \mathrm{H}_{2}\right)$ according to the Computational Hydrogen Electrode (CHE) model (Nørskov et al., 2004).

Overpotential $(\eta)$ is regarded as a parameter to measure the intrinsic activities of a catalyst, which is determined by:

$$
\eta=1.23 \mathrm{~V}+\operatorname{MAX}\left(\Delta \mathrm{G}_{1}, \Delta \mathrm{G}_{2}, \Delta \mathrm{G}_{3}, \Delta \mathrm{G}_{4}\right) / \mathrm{e}
$$

where $\Delta G_{1}, \Delta G_{2}, \Delta G_{3}$, and $\Delta G_{4}$ stand for the reaction free energy of four elementary reaction steps of ORR.

The adsorption energy of absorbed specie $x$ on the surface, $E_{a d-x}$, was calculated by:

$$
\mathrm{E}_{\mathrm{ad}-\mathrm{x}}=\mathrm{E}_{\mathrm{t}}-\mathrm{E}_{0}-\mathrm{E}_{\mathrm{x}}
$$

where $E_{t}, E_{0}$, and $E_{x}$ are the total energy of the adsorbed structure, the energy of the isolated GR structure, and the energy of absorbed species, respectively.

\section{RESULTS}

\section{Protonation in Acid Medium}

In acidic medium, there are a large number of protons and acid anions, which may affect the ORR and result in adverse effect to the catalytic activity of heteroatom doped graphene. To address the effect of protons on the catalytic activity of N-GR, the adsorption energy of protons and the ORR overpotential for the protonated N-GR were calculated. The proton adsorption energy was calculated at four different sites, shown as Figure 2, according to the previous work (Liu et al., 2010; Li et al., 2014; Guo et al., 2016). In order to make a comparison with the alkaline medium, adsorption energy of the hydroxyl was also calculated. As shown in Figure 2, both the proton and hydroxyl prefer to adsorb on site 1, the nitrogen atom, with the adsorption energies of -1.23 and $-2.51 \mathrm{eV}$, respectively. Thus, whether in acidic medium or alkaline medium, the nitrogen dopant at the edge of the graphene is easily protonated or terminated with hydroxyl.

In ORR, adsorption of $\mathrm{O}_{2}$ on the catalyst surface is a pivotal step. In the following calculations, the $\mathrm{N}-\mathrm{GR} / \mathrm{H}$ or $\mathrm{N}-\mathrm{GR} / \mathrm{OH}$ stands for one proton or hydroxyl adsorbed on the site 1 , nitrogen atom (Figure 2). We explored the adsorption of $\mathrm{O}_{2}$ on the surface of $\mathrm{N}-\mathrm{GR}, \mathrm{N}-\mathrm{GR} / \mathrm{H}$, and N-GR/OH. There are two types of adsorption for the $\mathrm{O}_{2}$ on N-GR, end-on adsorption (Figure 3A), and side-on adsorption (Figures 3B,C), which are crucial to the 


\section{A}
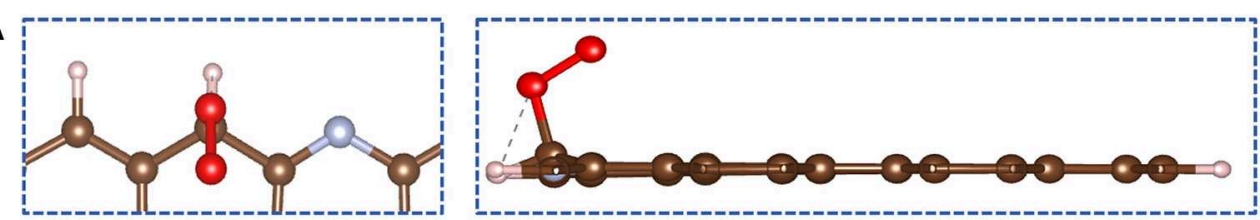

B

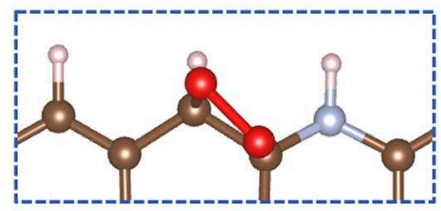

C

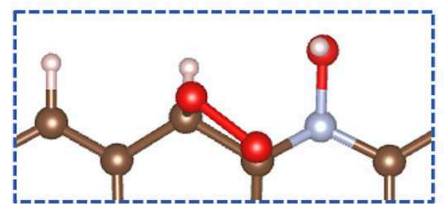

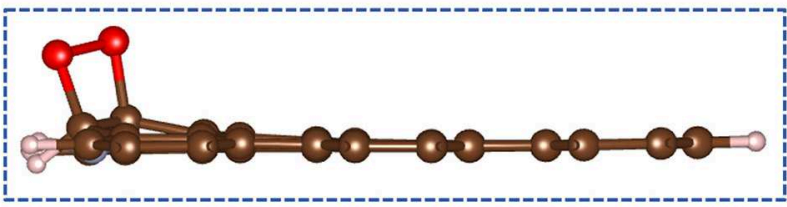

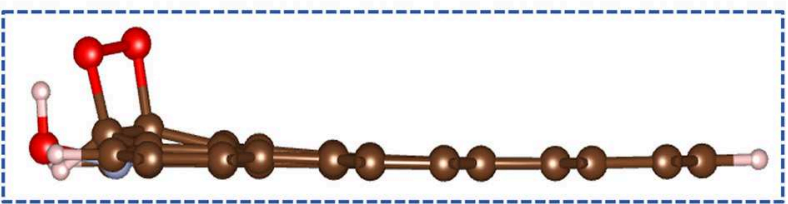

FIGURE 3 | The most stable structures for (A) N-GR, (B) N-GR/H, and (C) N-GR/OH when adsorbing $\mathrm{O}_{2}$. The left panels are top view and the right ones are side view.
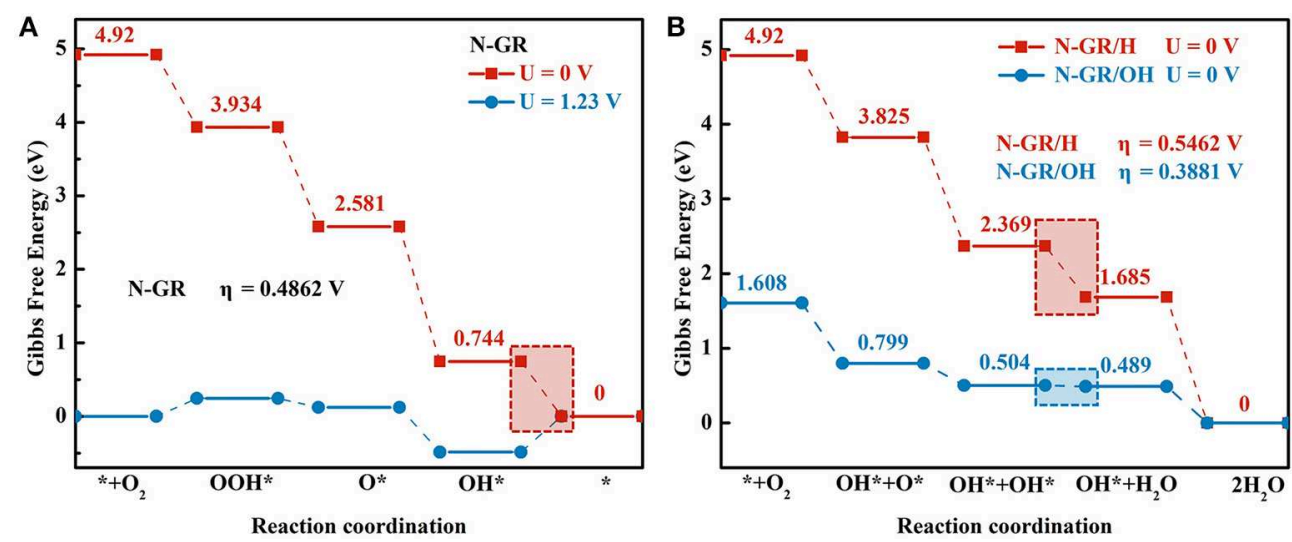

FIGURE 4 | The reaction Gibbs free energy diagrams of four elementary reaction steps of ORR on (A) N-GR and (B) N-GR/H and N-GR/OH. The shadow box highlights the rate-determining step.

subsequent reaction path for the ORR. The adsorption energies of $\mathrm{O}_{2}$ on N-GR, N-GR/H, and N-GR/OH are listed in Table $\mathbf{1}$ and S-Table 1. According to the calculated adsorption energy, $\mathrm{O}_{2}$ prefers to adsorb on N-GR with end-on mode, but with sideon mode on $\mathrm{N}-\mathrm{GR} / \mathrm{H}$ or $\mathrm{N}-\mathrm{GR} / \mathrm{OH}$. The end-on adsorption on $\mathrm{N}$-GR is $\sim 0.11 \mathrm{eV}$ higher than that of the side-on adsorption on $\mathrm{N}-\mathrm{GR} / \mathrm{H}$ and $\mathrm{N}-\mathrm{GR} / \mathrm{OH}$. These results indicate that the acidic medium not only changes the adsorption mode of the $\mathrm{O}_{2}$ on $\mathrm{N}-\mathrm{GR}$, but also makes the adsorption harder than in alkaline medium. The relatively larger adsorption energy of $\mathrm{O}_{2}$ is unfavorable to the ORR. Therefore, the acidic medium is unfavorable to the adsorption of $\mathrm{O}_{2}$, and suppress the catalytic activity of N-GR.

Besides the effect of protonation on the first reaction step of ORR and $\mathrm{O}_{2}$ adsorption, we also explored the effects of protonation on the overall ORR on N-GR. The reaction free
TABLE 1 | The adsorption energy of $\mathrm{O}_{2}$ on different doped GRs by end-on mode and side-on mode. (Unit/eV).

\begin{tabular}{lcc}
\hline Catalysts & End-on mode & Side-on mode \\
\hline $\mathrm{N}-\mathrm{GR}$ & 0.27 & 0.82 \\
$\mathrm{~N}-\mathrm{GR} / \mathrm{H}$ & 0.83 & 0.40 \\
$\mathrm{~N}-\mathrm{GR} / \mathrm{OH}$ & 0.43 & 0.29 \\
\hline
\end{tabular}

energy of the sub-reaction and the overpotential of the whole ORR on N-GR, N-GR/H, and N-GR/OH were calculated. We chose the most active site in each structure by testing all the potential active sites near the doped heteroatom. The reaction free energy of the sub-reaction on N-GR is shown in Figure 4A. The ORR proceeds with the associate mechanism, and the ratelimiting step is the desorption of ${ }^{*} \mathrm{OH}$ to form $\mathrm{H}_{2} \mathrm{O}$. The 


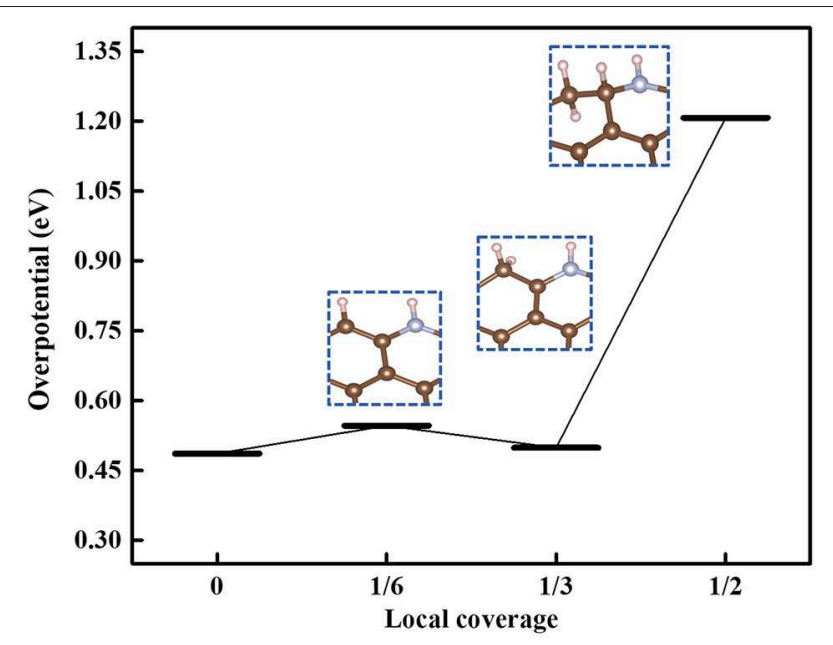

FIGURE 5 | The overpotential of ORR at different local coverage rate. The models in the blue dotted frame are the best adsorption configuration under this local coverage.

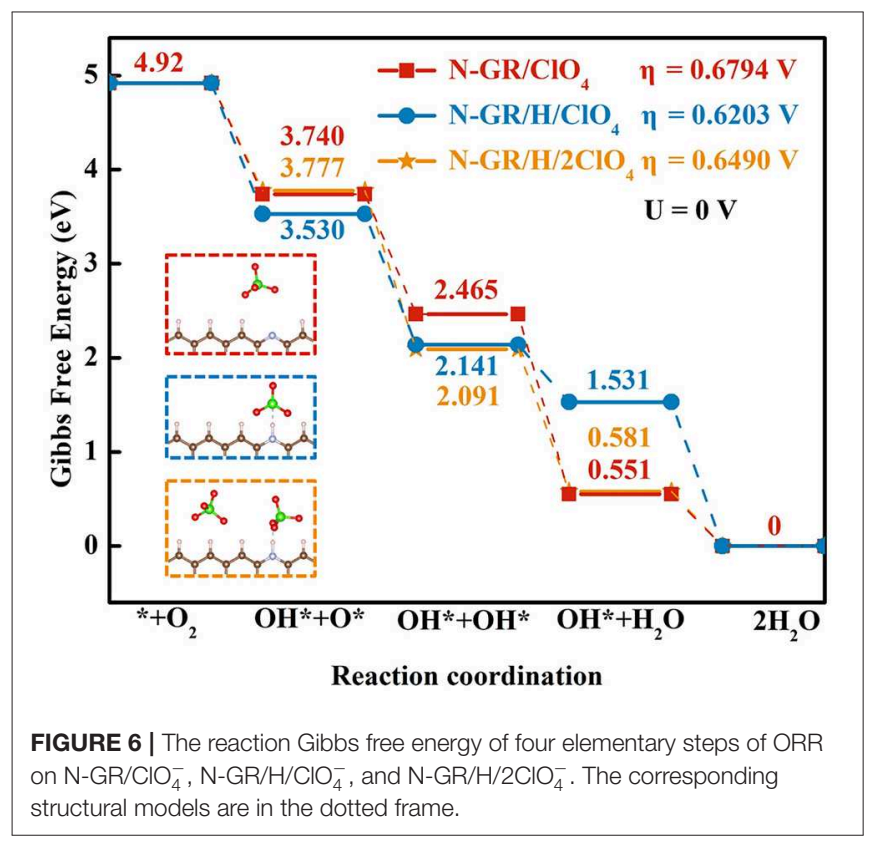

overpotential for the ORR is $0.49 \mathrm{~V}$. On the contrary, as shown in the reaction free energy diagram of ORR on $\mathrm{N}-\mathrm{GR} / \mathrm{H}$ and $\mathrm{N}$ GR/OH (Figure 4B), the ORR follows dissociative pathway. The reaction rate-limiting step is changed to the desorption of the first ${ }^{*} \mathrm{OH}$ to form $\mathrm{H}_{2} \mathrm{O}$, and the overpotentials for ORR on $\mathrm{N}$ $\mathrm{GR} / \mathrm{H}$ and $\mathrm{N}-\mathrm{GR} / \mathrm{OH}$ are $0.55 \mathrm{~V}$ and $0.39 \mathrm{~V}$, respectively. Thus, the acidic medium would change the ORR mechanism on N-GR and the reaction rate-limiting step. As a result, the overpotential of ORR in acidic environments significantly increases compared with that in alkaline medium.

In acidic medium, the protons are adsorbed not only at $\mathrm{N}$ atoms but also at other sites in N-GR. We therefore studied the effect of the hydrogen coverage rate over N-GR on the ORR catalytic activities. The local coverage rate is defined as $n / 6$ monolayer (ML), where $n$ is the number of adsorbed hydrogen, and 6 represents the possible active sites near the dopant $\mathrm{N}$. The overpotentials were calculated for the most stable adsorbed structures of N-GR with different local coverage rate values $0,1 / 6 \mathrm{ML}, 1 / 3 \mathrm{ML}$, and $1 / 2 \mathrm{ML}$, and the results are shown in Figure 5. With increasing the local coverage rate, the overpotential increases. Therefore, in acidic medium, with the development of the protonation, the ORR catalytic activity becomes worse. It should be noted that in Figure 5, the overpotential at the local coverage of $1 / 3 \mathrm{ML}$ is lower than that of $1 / 6 \mathrm{ML}$, but the hydrogen adsorption energy on the N-GR with the local coverage rate of $1 / 3 \mathrm{ML}\left(\mathrm{E}_{\mathrm{ad}-\mathrm{H}}=-1.11 \mathrm{eV}\right)$ is higher than that on the $\mathrm{N}$ GR with the local coverage rate of $1 / 6 \mathrm{ML}$ and $1 / 2 \mathrm{ML}$ (with the value of $\mathrm{E}_{\mathrm{ad}-\mathrm{H}}=-1.26 \mathrm{eV}$ and $-1.40 \mathrm{eV}$, respectively). Evidently, with increasing the protonation, the local coverage rate would change from $1 / 6 \mathrm{ML}$ to $1 / 2 \mathrm{ML}$ quickly. Therefore, the ORR catalytic activity of N-GR decreases with increasing the protonation.

\section{Adsorption of Anions in Acid Medium}

As mentioned above, the acid anion is one of the main factors that affect the catalytic activity of N-GR in acidic medium. The perchloric acid and sulfuric acid are the two most common acids used as acidic electrolytes in fuel cells (Mamtani et al., 2018; Sun et al., 2018; Mun et al., 2019; Zhang L. et al., 2019). In order to study the effects of acid anions on the catalytic activity of N-GR, we introduced acid anions (including $\mathrm{ClO}_{4}^{-}, \mathrm{SO}_{4}^{2-}$, and $\mathrm{HSO}_{4}^{-}$) near the N-GR structures, and calculated the overpotential and reaction pathways. When the acid anions adsorb on the N-GR, it could be located at different positions near the N-GR. It was found that the acid anions preferred to aggregate near the edge of the N-GR not the basal plane of the N-GR, the adsorption structures are shown as S-Figures 1G-L.

Figure 6 shows the reaction free energy diagram of ORR on the structures with one perchlorate $\left(\mathrm{N}-\mathrm{GR} / \mathrm{ClO}_{4}^{-}\right)$, one perchlorate and one adsorbed $\mathrm{H}\left(\mathrm{N}-\mathrm{GR} / \mathrm{H} / \mathrm{ClO}_{4}^{-}\right)$, and two perchlorates and one adsorbed $\mathrm{H}\left(\mathrm{N}-\mathrm{GR} / \mathrm{H} / 2 \mathrm{ClO}_{4}^{-}\right)$(the inset in Figure 6), the total energy of the adsorbed intermediate on these structures are listed in S-Table 2. The overpotentials of ORR on $\mathrm{N}-\mathrm{GR} / \mathrm{ClO}_{4}^{-}, \mathrm{N}-\mathrm{GR} / \mathrm{H} / \mathrm{ClO}_{4}^{-}$, and $\mathrm{N}-\mathrm{GR} / \mathrm{H} / 2 \mathrm{ClO}_{4}^{-}$are 0.68 , 0.62 , and $0.65 \mathrm{~V}$, respectively. These values are all higher than the overpotential of ORR on N-GR/H. Thus, the existing of acid anions on the N-GR surface is detrimental to the ORR catalytic activity. In addition, the effect of adsorption of perchlorate was stronger than protonation because the overpotential of ORR on $\mathrm{N}-\mathrm{GR} / \mathrm{ClO}_{4}^{-}$is a little bit higher than that on $\mathrm{N}$ $\mathrm{GR} / \mathrm{H} / \mathrm{ClO}_{4}^{-}$. As the number of perchlorates increases to two, the overpotential of ORR increases to $0.65 \mathrm{~V}$. It indicated that the more aggregation of the perchlorate near the $\mathrm{N}$ GR, the more adverse effects on the ORR catalytic activity. Similar effect was also found for $\mathrm{HSO}_{4}^{-}$and $\mathrm{SO}_{4}^{2-}$ on the ORR catalytic activity of N-GR. The overpotentials of ORR for N$\mathrm{GR} / \mathrm{HSO}_{4}^{-}, \mathrm{N}-\mathrm{GR} / \mathrm{SO}_{4}^{2-}$ were calculated to be 0.50 and $1.42 \mathrm{~V}$, respectively (Figure 7). The effect of $\mathrm{SO}_{4}^{2-}$ on ORR catalytic 
activity is more adverse than that of $\mathrm{HSO}_{4}^{-}$, and much more adverse than that of $\mathrm{ClO}_{4}^{-}$. Therefore, the adsorption of acid anions on N-GR could be considered as one of the main factors for the degeneration of the ORR catalytic activity in acidic medium.

\section{DISCUSSION}

The unfavorable effects of protonation and acid anions on ORR catalytic activity of N-GR can be ascribed to charge redistribution at the active sites generated by the adsorbed hydrogen or acid anions. Figure 8 shows differential charge density distribution of hydrogen or acid anion adsorbed N-GR, which is calculated by the charge density distribution on the adsorption N-GR minus that on the un-adsorption structures. The charges on the active sites C- 1 and C-2 are changed due to the adsorption of hydrogen or acid anions, which could influence the adsorption of $\mathrm{O}_{2}$, desorption of ${ }^{*} \mathrm{OH}$ in the sub-reaction of ORR. For example, on $\mathrm{N}-\mathrm{GR} / \mathrm{H}$, the positive charges decrease due to the electron transfer from the adsorbed $\mathrm{H}$ (Figure 8B), which is unfavorable to the adsorption of $\mathrm{O}_{2}$ at $\mathrm{C}-1$ and $\mathrm{C}-2$ sites with side-on mode, as shown in Figure 3B and S-Figure 2B. Moreover, after the breakage of $\mathrm{O}-\mathrm{O}$ bond to form two adsorbed ${ }^{*} \mathrm{OH}$ at $\mathrm{C}-1$ and $\mathrm{C}-2$, one of the ${ }^{*} \mathrm{OH}$ is unfavorable to proceed with desorption of formed $\mathrm{H}_{2} \mathrm{O}$, which acts as the reaction rate-limiting step in ORR (Figure 4B). On N-GR/ $\mathrm{ClO}_{4}^{-}$, the $\mathrm{C}-1$ and $\mathrm{C}-2$ possess more positive charges (0.63 and 0.07 ) because of the induced polarization between the $\mathrm{C}$ atom and the $\mathrm{O}$ atom from the $\mathrm{ClO}_{4}^{-}$(Figure 8D). The excessive positive charges on $\mathrm{C}-1$ and $\mathrm{C}-2$ are favorable to the adsorption of $\mathrm{O}_{2}$ (S-Figure 2D), but detrimental to the desorption of ${ }^{*} \mathrm{OH}$. On $\mathrm{N}-\mathrm{GR} / \mathrm{H} / \mathrm{SO}_{4}^{2-}, \mathrm{C}-1$ and $\mathrm{C}-2$ possess charges with the value of 0.44 and 0.03 (Figure $8 \mathrm{~F}$ ), which is favorable to the adsorption of $\mathrm{O}_{2}$, but detrimental to the desorption of ${ }^{*} \mathrm{OH}$ due to the synergistic effect of high positive charge on adsorbed site and the electrostatic repulsive force from the oxygen atom

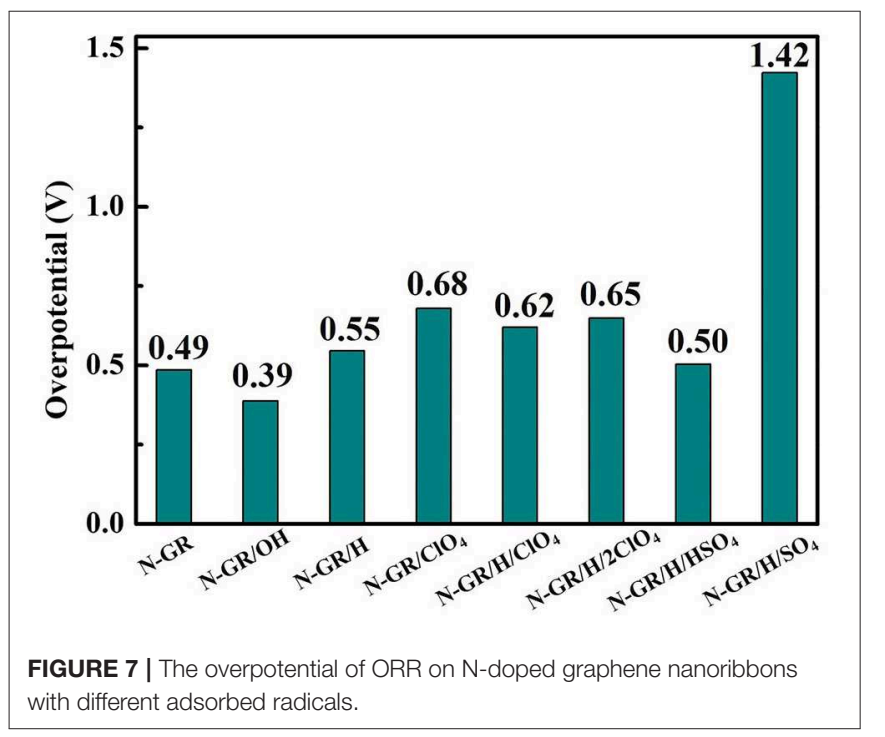

in the $\mathrm{SO}_{4}^{2-}$. On $\mathrm{N}-\mathrm{GR} / \mathrm{OH}$, the $\mathrm{C}-1$ and $\mathrm{C}-2$ possess proper quantity of positive charges (Figure $\mathbf{8 C}$ ), which is moderate for both adsorption of $\mathrm{O}_{2}$ (S-Figure 2C) and desorption of ${ }^{*} \mathrm{OH}$. Therefore, the desired active sites should not only be favorable to the adsorption of $\mathrm{O}_{2}$, but also advantageous to the desorption of ${ }^{*} \mathrm{OH}$ conforming to the Sabatier principle (Lin et al., 2017).

Besides the nitrogen doped graphene nanoribbon (N-GR), we also studied the influence of protons, hydroxyls and acid anions on the ORR catalytic performance of other heteroatom doped graphene nanoribbons, such as B-GR and P-GR. The ORR overpotentials corresponding to different doped structures are listed in S-Table 3. Similar to N dopants, the protonation and acid anions are also detrimental to the ORR catalytic activity for the B- and P-doped graphene nanoribbons. Protonation shows an obvious adverse effect on the catalytic activity of P-GR. However, the adsorption of hydroxyl radical on the B-GN is favorable to the catalytic activity. For all the doped structures, sulphuric acid shows more adverse effect on the ORR catalytic activity than the perchloric acid. Thus, the adverse effects of protonation and acid anions are common to the heteroatom doped graphene nanoribbon.

The effects of proton and acidic anions on the catalytic activity of doped graphene in our simulation work are highly consistent with published experimental works (Xue et al., 2018; Yang et al., 2019). For instance, the nitrogen-doped carbon catalysts were characterized with the XPS spectra before and after the potential cycling stability test in acid and alkaline electrolytes by $\mathrm{Li}$ et al., they found there were more pyridinic nitrogen changed to graphitic nitrogen in acidic electrolyte than in alkaline electrolyte ( $\mathrm{Li}$ et al., 2010). The change of $\mathrm{N}$ from pyridinic to graphitic form could be ascribed to the protonation of pyridinic nitrogen. Yang et al. also mentioned in their review work that the active sites of the catalysts would be blocked by the adsorbed anions in acidic electrolyte (Yang et al., 2019). Besides the detrimental effects of acidic anion on the work electrode catalysts, the anion would also interact with the counter electrode, which may be one of the reasons of the inferior catalytic activities of catalysts in acidic environment (Zhang et al., 2014).

To reduce, even eliminate the detrimental effects of protonation and acid anions on the ORR catalytic activity of doped graphene, an effective way is to prevent the protonation and the acid anions, such that their influence on the charge distribution of the active sites can be eliminated. To achieve this goal, we propose several strategies as follows. (1) The dopant atom position could be changed to make the protonation not easily processed. For example, the dopant atom could be located at the intrinsic defects (such as Stone Wales defects, Vacancy defects) not at the edge of the graphene. (2) Particular radical could be added into the electrolyte solution, which could terminate the protonated sites but not change the charge distribution of the active sites too much. (3) The acid anions can be segregated from the active sites as much as possible. For example, design multi-scale hierarchical porous structure (Liu et al., 2019; Yang et al., 
A

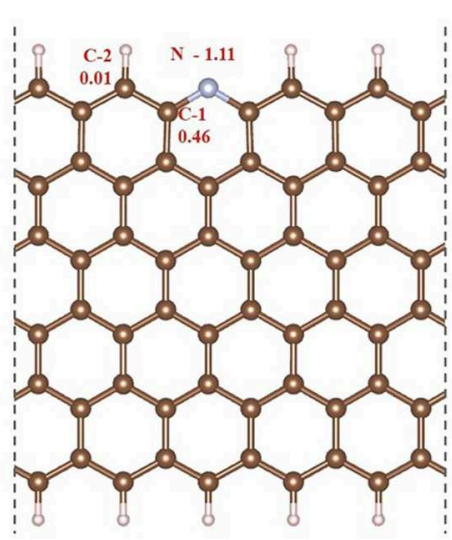

B

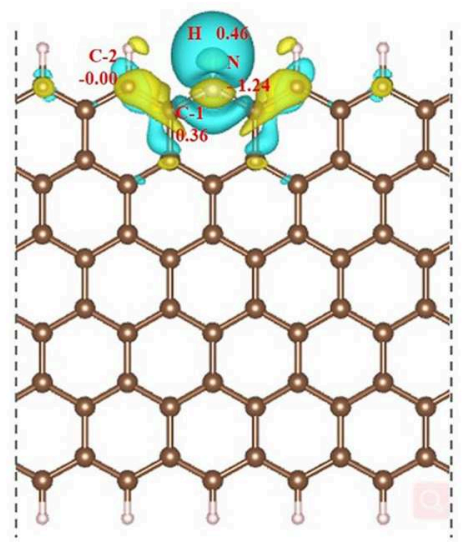

C

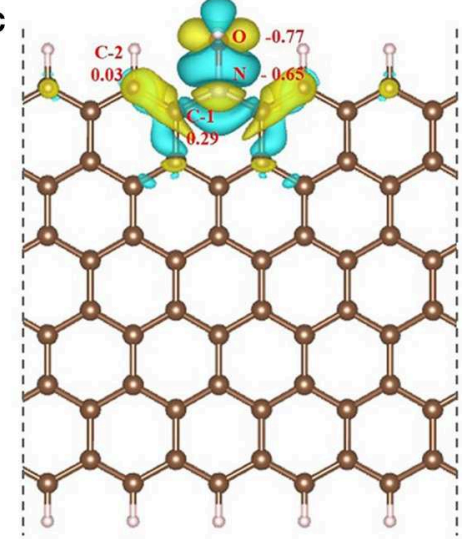

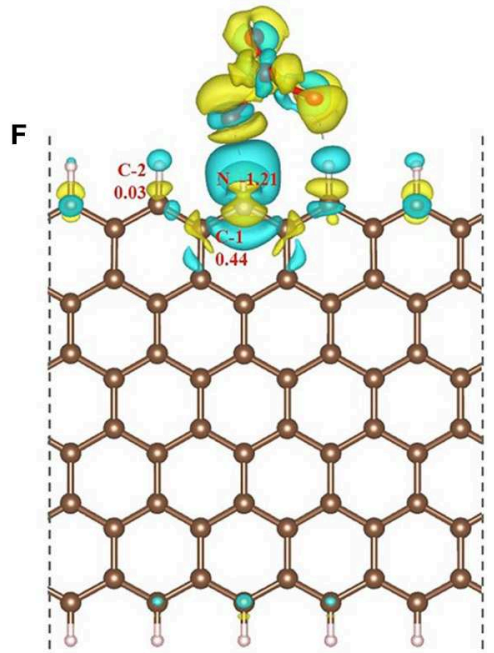

FIGURE 8 | Differential charge density distributions (between absorbed and un-adsorbed structures) and Bader charge of the adsorption sites on (A) N-GR, (B) $\mathrm{N}-\mathrm{GR} / \mathrm{H}$, (C) N-GR/OH, (D) N-GR/ClO ${ }_{4}^{-}$, (E) N-GR/H/CIO ${ }_{4}^{-}$, and (F) N-GR/H/SO ${ }_{4}^{2-}$. The Yellow represents the electron accumulation area, and the blue represents the electron loss area. The isosurface value is set to $2.5 \times 10^{-3} \mathrm{e} / \mathrm{Bohr}^{3}$.

2019; Zhang L. et al., 2019), which could block the acid anions approaching to the reactive sites but not influence the transfer of reactants, products and reaction intermediates. (4) Defective graphene structure could be designed to make the active sites locate at the central part, not the edge of the graphene, because the acid anions prefer to aggregate at the edge of the graphene.

\section{CONCLUSIONS}

We have systematically explored the possible reasons of the receding catalytic activity of doped graphene structures in acidic medium with DFT calculations. The results indicate that the protonation and acid anions show adverse effects on the catalytic performance of doped graphene. The adsorption of $\mathrm{H}$ on the dopant atom could change the ORR pathways and increase the ORR overpotential. The adsorption of acid anions near the edge of the doped graphene redistributes the charge on the active site, which influences the adsorption of $\mathrm{O}_{2}$ and desorption of ${ }^{*} \mathrm{OH}$ in the ORR process and therefore increases the overpotential, consequently suppresses the catalytic performance. Reducing or avoiding the protonation and adverse influence of acid anions could be a promising design strategy to enhance the ORR catalytic activity of doped carbon based catalysts in acid environment. Our findings provide hints and a direction to guide the rational design of highly efficient heteroatom-doped carbon based catalysts for ORR in acid medium.

\section{DATA AVAILABILITY STATEMENT}

All datasets generated for this study are included in the article/Supplementary Material.

\section{AUTHOR CONTRIBUTIONS}

JM did the calculations and wrote the paper. LG and DL reviewed and revised the paper. YS, BL, JZ, and DS joined discussion of the paper. $\mathrm{LZ}$ and $\mathrm{ZX}$ designed the work, reviewed and revised the paper. 


\section{FUNDING}

This work was financially supported by the National Key Research and Development Program of China (2017YFA0206500), National Natural Science Foundation of China (51732002, 51704243, 51973174, and 5164205), Distinguished Scientist Program at BUCT (buctylkxj02), the Fundamental Research Funds for the Central Universities (3102019ZD0402), and US National Science Foundation

\section{REFERENCES}

Dai, L., Xue, Y., Qu, L., Choi, H. J., and Baek, J. B. (2015). Metal-free catalysts for oxygen reduction reaction. Chem. Rev. 115, 4823-4892. doi: 10.1021/cr5003563

Debe, M. (2012). Electrocatalyst approaches and challenges for automotive fuel cells. Nature 486, 43-51. doi: 10.1038/nature11115

Deng, D., Novoselov, K., Fu, Q., Zheng, N., Tian, Z., and Bao, X. (2016). Catalysis with two-dimensional materials and their heterostructures. Nat. Nanotechnol. 11, 218-230. doi: 10.1038/nnano.2015.340

Gong, K., Du, F., Xia, Z., Durstock, M., and Dai, L. (2009). Nitrogen-doped carbon nanotube arrays with high electrocatalytic activity for oxygen reduction. Science 323, 760-764. doi: 10.1126/science.1168049

Guo, D., Shibuya, R., Akiba, C., Saji, S., Kondo, T., and Nakamura, J. (2016). Active sites of nitrogen-doped carbon materials for oxygen reduction reaction clarified using model catalysts. Science 351, 361-365. doi: 10.1126/science.aad0832

He, W., Wang, Y., Jiang, C., and Lu, L. (2016). Structural effects of a carbon matrix in non-precious metal $\mathrm{O}_{2}$-reduction electrocatalysts. Chem. Soc. Rev. 45, 2396-2409. doi: 10.1039/C5CS00665A

Hu, C., and Dai, L. (2019). Doping of carbon materials for metal-free electrocatalysis. Adv. Mater. 31:1804672. doi: 10.1002/adma.201804672

Ji, Y., Dong, H., Liu, C., and Li, Y. (2018). The progress of metal-free catalysts for the oxygen reduction reaction based on theoretical simulations. J. Mater. Chem. A. 6, 13489-13508. doi: 10.1039/C8TA02985G

Jiang, D., Sumpter, B. G., and Dai, S. (2007). Unique chemical reactivity of a graphene nanoribbon's zigzag edge. J. Chem. Phys. 126:134701. doi: $10.1063 / 1.2715558$

Jiang, H., Gu, J., Zheng, X., Liu, M., Qiu, X., Wang, L., et al. (2019). Defect-rich and ultrathin $\mathrm{N}$ doped carbon nanosheets as advanced trifunctional metal-free electrocatalysts for the ORR, OER and HER. Energ. Environ. Sci. 12, 322-333. doi: 10.1039/C8EE03276A

Kresse, G., and Furthmüller, J. (1996a). Efficiency of ab-initio total energy calculations for metals and semiconductors using a plane-wave basis set. Comp. Mater. Sci. 6, 15-50. doi: 10.1016/0927-0256(96)00008-0

Kresse, G., and Furthmüller, J. (1996b). Efficient iterative schemes for ab initio total-energy calculations using a plane-wave basis set. Phys. Rev. B. 54:11169. doi: 10.1103/PhysRevB.54.11169

Kresse, G., and Joubert, D. (1999). From ultrasoft pseudopotentials to the projector augmented-wave method. Phys. Rev. B. 59:1758. doi: 10.1103/PhysRevB.59.1758

Kulkarni, A., Siahrostami, S., Patel, A., and Nørskov, J. K. (2018). Understanding catalytic activity trends in the oxygen reduction reaction. Chem. Rev. 118, 2302-2312. doi: 10.1021/acs.chemrev.7b00488

Li, F., Shu, H., Liu, X., Shi, Z., Liang, P., and Chen, X. (2017). Electrocatalytic activity and design principles of heteroatom-doped graphene catalysts for oxygen-reduction reaction. J. Phys. Chem. C. 121, 14434-14442. doi: 10.1021/acs.jpcc.7b03093

Li, M., Zhang, L., Xu, Q., Niu, J., and Xia, Z. (2014). N-doped graphene as catalysts for oxygen reduction and oxygen evolution reactions: theoretical considerations. J. Catal. 314, 66-72. doi: 10.1016/j.jcat.2014.03.011

Li, X., Liu, G., and Popov, B. N. (2010). Activity and stability of non-precious metal catalysts for oxygen reduction in acid and alkaline electrolytes. J. Power Sour. 195, 6373-6378. doi: 10.1016/j.jpowsour.2010.04.019

Lin, C. Y., Zhang, L., Zhao, Z., and Xia, Z. (2017). Design principles for covalent organic frameworks as efficient electrocatalysts in clean
(1561886 and 1662288) for the support of this research. Thanks to the Sino-Foreign Cooperative Training Project of BUCT.

\section{SUPPLEMENTARY MATERIAL}

The Supplementary Material for this article can be found online at: https://www.frontiersin.org/articles/10.3389/fmats. 2019.00294/full\#supplementary-material

energy conversion and green oxidizer production. Adv. Mater. 29:1606635. doi: 10.1002/adma.201606635

Liu, D., Dai, L., Lin, X., Chen, J. F., Zhang, J., Feng, X., et al. (2019). Chemical approaches to carbon-based metal-free catalysts. Adv. Mater. 31:1804863. doi: 10.1002/adma.201804863

Liu, G., Li, X., Ganesan, P., and Popov, B. N. (2010). Studies of oxygen reduction reaction active sites and stability of nitrogen-modified carbon composite catalysts for PEM fuel cells. Electrochim. Acta. 55, 2853-2858. doi: 10.1016/j.electacta.2009.12.055

Liu, X., and Dai, L. (2016). Carbon-based metal-free catalysts. Nat. Rev. Mater. 1:16064. doi: 10.1038/natrevmats.2016.64

Lu, Z., Wang, B., Hu, Y., Liu, W., Zhao, Y., Yang, R., et al. (2019). An isolated zinc-cobalt atomic pair for highly active and durable oxygen reduction. Angew. Chem. Int. Edit. 131, 2648-2652. doi: 10.1002/ange.201810175

Mamtani, K., Jain, D., Dogu, D., Gustin, V., Gunduz, S., Co, A. C., and Ozkan, U. S. (2018). Insights into oxygen reduction reaction (ORR) and oxygen evolution reaction (OER) active sites for nitrogen-doped carbon nanostructures $(\mathrm{CNx})$ in acidic media. Appl. Catal. B Environ. 220, 88-97. doi: 10.1016/j.apcatb.2017.07.086

Man, I. C., Su, H., Calle-Vallejo, F., Hansen, H., Martínez, J., Inoglu, N., et al. (2011). Universality in oxygen evolution electrocatalysis on oxide surfaces. ChemCatChem 3, 1159-1165. doi: 10.1002/cctc.201000397

Mun, Y., Lee, S., Kim, K., Kim, S., Lee, S., Han, J. W., and et al. (2019). Versatile strategy for tuning ORR activity of a single Fe-N4 site by controlling electronwithdrawing/donating properties of a carbon plane. J. Am. Chem. Soc. 141, 6254-6262. doi: 10.1021/jacs.8b13543

Nørskov, J. K., Rossmeisl, J., Logadottir, A., Lindqvist, L. R. K. J., Kitchin, J. R., Bligaard, T., and et al. (2004). Origin of the overpotential for oxygen reduction at a fuel-cell cathode. J. Phys. Chem. B. 108, 17886-17892. doi: 10.1021/jp047349j

Perdew, J. P., Burke, K., and Ernzerhof, M. (1996). Generalized gradient approximation made simple. Phys. Rev. Lett. 77:3865. doi: 10.1103/PhysRevLett.77.3865

Shao, M., Chang, Q., Dodelet, J., and Chenitz, R. (2016). Recent advances in electrocatalysts for oxygen reduction reaction. Chem. Rev. 116, 3594-3657. doi: 10.1021/acs.chemrev.5b00462

Stephens, I., Rossmeisl, J., and Chorkendorff, I. (2016). Toward sustainable fuel cells. Science 354, 1378-1379. doi: 10.1126/science.aal3303

Sun, J., Lowe, S. E., Zhang, L., Wang, Y., Pang, K., Wang, Y., et al. (2018). Ultrathin Nitrogen-Doped Holey Carbon@ graphene bifunctional electrocatalyst for oxygen reduction and evolution reactions in alkaline and acidic media. Angew. Chem. Int. Edit. 130, 16749-16753. doi: 10.1002/ange.2018 11573

Wan, K., Yu, Z., Li, X., Liu, M., Yang, G., and Piao, J. (2015). pH effect on electrochemistry of nitrogen-doped carbon catalyst for oxygen reduction reaction. ACS Catal. 5, 4325-4332. doi: 10.1021/acscatal.5b01089

Wang, N., Li, T., Song, Y., Liu, J., and Wang, F. (2018b). Metal-free nitrogen-doped porous carbons derived from pomelo peel treated by hypersaline environments for oxygen reduction reaction. Carbon 130, 692-700. doi: 10.1016/j.carbon.2018.01.068

Wang, N., Lu, B., Li, L., Niu, W., Tang, Z., and Kang, X. (2018a). Graphitic nitrogen is responsible for oxygen electroreduction on nitrogen-doped carbons in alkaline electrolytes: Insights from activity attenuation studies and theoretical calculations. ACS Catal. 8, 6827-6836. doi: 10.1021/acscatal.8b00338 
Wu, J., Jin, C., Yang, Z., Tian, J., and Yang, R. (2015). Synthesis of phosphorusdoped carbon hollow spheres as efficient metal-free electrocatalysts for oxygen reduction. Carbon 82, 562-571. doi: 10.1016/j.carbon.2014.11.008

Xue, L., Li, Y., Liu, X., Liu, Q., Shang, J., and Duan, H. (2018). Zigzag carbon as efficient and stable oxygen reduction electrocatalyst for proton exchange membrane fuel cells. Nat. Commun. 9:3819. doi: 10.1038/s41467-01806279-x

Yang, L., Jiang, S., Zhao, Y., Zhu, L., Chen, S., Wang, X., et al. (2011). Borondoped carbon nanotubes as metal-free electrocatalysts for the oxygen reduction reaction. Angew. Chem. Int. Edit. 50, 7132-7135. doi: 10.1002/anie.2011 01287

Yang, L., Shui, J., Du, L., Shao, Y., and Liu, J., et al. (2019). Carbon-based metalfree ORR electrocatalysts for fuel cells: past, present, and future. Adv. Mater. 31:1804799. doi: 10.1002/adma.201804799

Yang, N., Li, L., Li, J., Ding, W., and Wei, Z. (2018). Modulating the oxygen reduction activity of heteroatom-doped carbon catalysts via the triple effect: charge, spin density and ligand effect. Chem. Sci. 9, 5795-5804. doi: 10.1039/C8SC01801D

Yang, N., Zheng, X., Li, L., Li, J., and Wei, Z. (2017). Influence of phosphorus configuration on electronic structure and oxygen reduction reactions of phosphorus-doped graphene. J. Phys. Chem. C. 121, 19321-19328. doi: 10.1021/acs.jpcc.7b06748

Zeradjanin, A. (2018). Is a major breakthrough in the oxygen electrocatalysis possible? Curr. Opin. Electrochem. 9, 214-223. doi: 10.1016/j.coelec.2018.04.006

Zhang, C., Mahmood, N., Yin, H., Liu, F., and Hou, Y. (2013). Synthesis of phosphorus-doped graphene and its multifunctional applications for oxygen reduction reaction and lithium ion batteries. Adv. Mater. 25, 4932-4937. doi: 10.1002/adma.201301870

Zhang, G., Jia, Y., Zhang, C., Xiong, X., Sun, K., Chen, R., and et al. (2019). A general route via formamide condensation to prepare atomically dispersed metal-nitrogen-carbon electrocatalysts for energy technologies. Energ. Environ. Sci. 12, 1317-1325. doi: 10.1039/C9EE00162J

Zhang, L., Lin, C., Zhang, D., Gong, L., Zhu, Y., and Zhao, Z. (2019). Guiding principles for designing highly efficient metal-free carbon catalysts. Adv. Mater. 31:1805252. doi: 10.1002/adma.201805252

Zhang, X., Yu, S., Zheng, W., and Liu, P. (2014). Stability of Pt near surface alloys under electrochemical conditions: a model study. Phys. Chem. Chem. Phys. 16, 16615-16622. doi: 10.1039/C4CP01942C

Zhao, S., Wang, D. W., Amal, R., and Dai, L. (2019). Carbon-based metal-free catalysts for key reactions involved in energy conversion and storage. $A d v$. Mater. 31:1801526. doi: 10.1002/adma.201801526

Zhao, Z., Li, M., Zhang, L., Dai, L., and Xia, Z. (2015). Design principles for heteroatom-doped carbon nanomaterials as highly efficient catalysts for fuel cells and metal-air batteries. Adv. Mater. 27, 6834-6840. doi: 10.1002/adma.201503211

Zhu, Y., Zhang, D., Gong, L., Zhang, L., and Xia, Z. (2019). Catalytic activity origin and design principles of graphitic carbon nitride electrocatalysts for hydrogen evolution. Front. Mater. 6:16. doi: 10.3389/fmats.2019.00016

Conflict of Interest: The authors declare that the research was conducted in the absence of any commercial or financial relationships that could be construed as a potential conflict of interest.

Copyright (c) 2019 Ma, Gong, Shen, Sun, Liu, Zhang, Liu, Zhang and Xia. This is an open-access article distributed under the terms of the Creative Commons Attribution License (CC BY). The use, distribution or reproduction in other forums is permitted, provided the original author(s) and the copyright owner(s) are credited and that the original publication in this journal is cited, in accordance with accepted academic practice. No use, distribution or reproduction is permitted which does not comply with these terms. 